\title{
Quantitative Research on Gas Exchange Loss of Internal Combustion Engine
}

\author{
Jinguang Liang \\ Department of Machinery and Power Engineering, Yingkou Institute of Technology, Yingkou 115000, China
}

Keywords: internal combustion engine; intake loss; exhaust loss; gas exchange loss

\begin{abstract}
At present, there is no theoretical equation for quantitatively calculating gas exchange losses of an internal combustion engine. In order to accurately research on change rules of the gas exchange losses during the working process of internal combustion engines, the equation for quantitative calculating the gas exchange losses should be established. This work presents a concept of Newtonian ideal airflow and an energy equation for the Newtonian ideal airflow, based on theoretical analysis. Experimental researches were also conducted by employing a LJ491QE1 gasoline engine and the gas exchange losses of the engine were calculated quantitatively by using the energy equation for Newtonian ideal airflow. The results shows that the intake loss power of the naturally aspirated port fuel injection gasoline engine make up a higher percentage of its effective output power under low load engine operating states, and the exhaust loss power of the engine make up a higher percentage of its effective output power under high load engine operating states. The theory for calculating the gas exchange losses quantitatively presented in this paper has important meaning to further research on the actual circulation loss of the internal combustion engines.
\end{abstract}

\section{Introduction}

The gas exchange losses of internal combustion engines consist of the intake loss and exhaust loss. Current researches on the gas exchange processes of internal combustion engines are in the stage of computational fluid dynamics and the numerical simulation is the main research method, which use various related business software on computers. At present, there is no theoretical equation established for quantitatively calculating the gas exchange losses, so the accuracy of above research method cannot be measured.

For a gasoline engine, during the intake process, air flows through the air filter, the throttle, the intake manifold, the intake valves, the variable sections and the bending parts of the intake pipe and so on. The velocity and direction of the airflow are forced to change dramatically, resulting in the fluid particles collisions, causing the phenomena of vortexes, reattachments, secondary flows, and the flow separations, etc. Due to the viscous effect of airflow, severe friction and momentum exchange emerge between the fluid particles and prevent the fluid from moving on, that is root cause of the local resistance loss, which is a major part of the intake loss, and the corresponding flow state of the airflow is turbulence. In addition, the intake loss also includes the route loss due to wall friction of the intake pipe and viscous friction between the fluid layers, and the corresponding flow state of the airflow is laminar. The gas exchange losses include the above local resistance loss and route loss in the intake process along with the expansion loss and push out loss in the exhaust process which is one of the major factors that influence the efficiency of internal combustion engines. In order to accurately research on the change rules of the gas exchange losses during the working process of the internal combustion engines, the equation for quantitative calculating the gas exchange losses should be established.

\section{Energy equation for Newtonian ideal airflow}

\subsection{A concept of the Newton ideal airflow}

It is known to all that the continuum medium theory will no longer be suitable for use when the ratio between the mean free path of molecules and the feature size of object studied is greater than $1 \%{ }^{[1]}$. In the general case, the actual gas such as air meets the Euler continuity assumption and it can be treated as a continuous medium. All its flow parameters, such as temperature, pressure, velocity and so on can be regarded as a continuous function of time and space. Under the condition of higher temperature or lower pressure, as long as the pressure is not higher than $10 \mathrm{MPa}^{[2]}$, the state parameters of an actual gas such as air basically meets the state equation of ideal gas and it can be regarded as an ideal gas. The viscosity is an intrinsic property of an actual fluid, an actual fluid such as air is essentially a Newtonian fluid and it meets the law of Newton internal friction.

Given the above, the concept of Newton ideal airflow is defined that the actual gas flow such as the air flow meets the Euler continuity assumption and the state equation of ideal gas and the law of Newton internal friction at the same time.

\subsection{A detail derivation process of the energy equation for Newtonian ideal airflow}

Thermodynamic state parameters of the Newton's ideal airflow meet the state equation of ideal gas when the airflow is in the state of equilibrium. To determine the airflow state, in addition to the two independent thermodynamic state parameters of the absolute pressure $p$, the density $\rho$, and the 
thermodynamic temperature $T$, the parameter of velocity is needed to describe how fast the airflow moving relative to the reference state such as the state of atmospheric. Under normal conditions, the fluid velocity $V$ refers to the average fluid speed at the flow section in engineering application. When the airflow flows in a tube, its gravity and change of space position is very small, its gravitational potential energy is generally ignored in the energy analysis.

Figure 1 shows that an isochoric Newton ideal airflow of viscosity $\mu$ flows steadily in a tube of radius $r$ at low velocity $V$ (Mach number less than 0.3) and forms a laminar flow state. The energy of unit mass of airflow at the section A, upstream end of the tube, depends on the state parameters of the airflow at section $\mathrm{A}$, include the absolute pressure $p_{l}$, the thermodynamic temperature $T_{l}$ and the velocity $V$. For the intake and exhaust of the engine, the thermodynamic parameters $p_{l}$ and $T_{l}$ can be measured directly by test devices, the velocity $V$ can be calculated on the basis of the working state parameters of the engine (method for calculating the velocity $V$ and the gas constant $R$ can be found in the reference [3]), and the energy of the airflow at section $A$ equals to the energy loss of the airflow from its initial state, the state 1 at section $\mathrm{A}$, to its final state, the state 0 , which is the atmospheric state and also is the reference state. The initial and final states of the airflow have been defined, even if the process characteristics between the two states are unknown, according to the first law of thermodynamics, the energy loss of airflow at section A from the state 1 to the state 0 , the reference state, is a constant, no matter what the intermediate processes are.

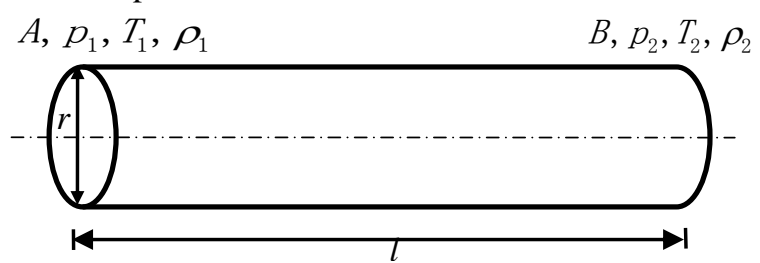

Figure 1: Isochoric flow model of Newtonian ideal airflow

Given the above, suppose that the unit mass of airflow of state 1 , the initial state, changes to its final state, the atmospheric state, experiencing the following two thermodynamic processes:

Process 1. The unit mass of airflow at the section $A$ of the tube whose initial state parameters are the thermodynamic temperature $T_{1}$, the absolute pressure $p_{l}$, the velocity $V_{l}$, the density $\rho_{l}$ and the viscosity $\mu$, experiences a fully developed laminar flow process of incompressible fluid to the section B of the tube, where the state parameters of the airflow are the thermodynamic temperature $T_{2}$, the absolute pressure $p_{2}$ and the density $\rho_{2}$. The pressure $p_{2}$ is the atmospheric pressure $p_{a}$, the viscosity $\mu$ and the specific volume of airflow remain constants during the whole process, and that is, the process is a constant volume heat release process of an open system.

Process 2. At the final state of process 1, the airflow arrived at the section $\mathrm{B}$ of the tube, and then, in the process 2 , the airflow at the section $\mathrm{B}$ experiences a constant pressure cooling process of a close system to the final state of process 2 , state 2 of the atmospheric state, which is the reference state of the airflow energy and the state parameters of the airflow are the thermodynamic temperature $T_{0}$, the absolute pressure $p_{0}$, the density $\rho_{a}$, and the final velocity of the airflow is zero.

Energy loss analysis of the process 1. The process 1 is a fully developed steady state laminar flow process of an incompressible fluid. Based on the first law of thermodynamics for an open system:

$$
\begin{aligned}
& \left(h_{1}+\frac{1}{2} V_{1}^{2}\right)-\left(h_{2}+\frac{1}{2} V_{2}^{2}\right) \\
& =h_{1}^{*}-h_{2}^{*}=w_{\mathrm{s}}^{1-2}-q_{1-2}
\end{aligned}
$$

Where the $h_{1}$ and $h_{2}$ represent respectively the enthalpy of unit mass of airflow at the section $\mathrm{A}$ and $\mathrm{B}$, the $h_{1}{ }^{*}$ and $h_{2}{ }^{*}$ represent respectively the stagnation enthalpy of unit mass of airflow at the section $\mathrm{A}$ and $\mathrm{B}$, the $q_{1-2}$ and $w_{s}^{1-2}$ represent respectively the heat release and shaft work output in the process 1 .

The process 1 is a constant volume heat release process of an open system, so the above equation would be written as:

$$
\begin{aligned}
& w_{\mathrm{s}}^{1-2}-q_{1-2}=h_{1}^{*}-h_{2}-\frac{1}{2} V_{1}^{2} \\
& =c_{\mathrm{p}}\left(T_{1}^{*}-T_{2}\right)-\frac{1}{2} V_{1}^{2}
\end{aligned}
$$

Inserting $p_{2}=p_{a}, \rho_{1}=\rho_{2}$, and $c_{\mathrm{p}}=\frac{k}{k-1} R$ to the above equation, gives the energy loss of unit mass of airflow in the process $1, e_{1-2}$ :

$$
\begin{aligned}
& \boldsymbol{e}_{1-2}=w_{\mathrm{s}}^{1-2}-q_{1-2}=R\left(T_{1}^{*}-T_{2}\right)-\frac{1}{2} V_{1}^{2} \\
& =\frac{k}{k-1} \frac{p_{1}^{*}-p_{2}}{\rho_{1}}-\frac{1}{2} V_{1}^{2} \\
& =\frac{k}{k-1} \frac{p_{\text {guage }}}{\rho_{1}}-\frac{1}{2} V_{1}^{2}
\end{aligned}
$$

Where the $p_{l}{ }^{*}$ and $p_{\text {guage }}$ represent respectively the stagnation pressure and gauge pressure of the airflow at section $\mathrm{A}$, the $k$ represents the specific heat ratio of the airflow.

In the process 1 , the airflow experiences a fully developed steady state laminar flow process of a viscous and incompressible fluid. Therefore, the shaft work $w_{s}^{l-2}$ is consumed by the wall friction of the tube and the viscous friction between the fluid layers, and released into the atmosphere in the form of heat at last. That is, during the course of the airflow flows from the section A to section B, the potential energy of the airflow at section A, caused by its pressure deviated from the reference pressure, the atmospheric pressure $p_{a}$, is eliminated because of the frictional work $w_{f}^{1-2}$ (equals to the shaft work $w_{s}{ }^{1-2}$ ) consumed by the fluid layers and the wall of the tube. The heat release in process $1 q_{1-2}$ depends on the properties of the process 1 and the airflow.

The frictional work $w_{f}^{1-2}$ is

$$
\begin{aligned}
& w_{\mathrm{f}}^{1-2}=w_{\mathrm{s}}^{1-2}=-\int_{1}^{2} v d p=\left(p_{1}^{*}-p_{2}\right) v \\
& =R\left(T_{1}^{*}-T_{2}\right)
\end{aligned}
$$


Where the $v$ represents the specific volume and the heat release in the process $1 q_{1-2}$ is

$$
\begin{aligned}
& q_{1-2}=u_{1}-u_{2}=\frac{1}{k-1} R\left(T_{1}^{*}-T_{2}\right) \\
& =\frac{1}{k-1}\left(p_{1}^{*}-p_{2}\right) v
\end{aligned}
$$

Energy loss analysis of the process 2. The final state of the airflow in the process 2 is the state of atmospheric, state 0 , according to the first law of thermodynamics for a close system, gives

$$
\begin{aligned}
& u_{2}-u_{0}+\frac{1}{2} V_{2}^{2}=c_{\mathrm{v}}\left(T_{2}-T_{0}\right)+\frac{1}{2} V_{2}^{2} \\
& =w_{\mathrm{v}}^{2-0}-q_{2-0}+\frac{1}{2} V_{2}^{2}
\end{aligned}
$$

Where the $c_{v}$ represents the specific heat at constant volume, $u_{2}$ and $u_{0}$ represent respectively the specific internal energy of the airflow at section B and the specific internal energy of the airflow in the state of atmospheric, the state 0 . The state 0 is the reference state, so $u_{0}=0$. The $w_{v}{ }^{2-0}$ represents the volume work to the unit mass of airflow by the external environment and the $q_{2-0}$ represents the heat released by the unit mass of airflow in the process 2 .

The process 2 is a cooling process of a close system under constant pressure, and the potential energy of the airflow at section B caused by its temperature deviated from the reference temperature, the atmospheric temperature, is eliminated because of the heat release $q_{2-0}$. The volume work in the process $2, \mathrm{~W}_{\mathrm{v}}{ }^{2-0}$, depends on the properties of the process and the airflow.

Inserting $p_{2}=p_{a}$, and $c_{\mathrm{v}}=\frac{R}{k-1}$ to the above equation, gives the energy loss of the unit mass of airflow during the course of process $2, e_{2-0}$ :

$$
\begin{aligned}
& e_{2-0}=w_{\mathrm{v}}^{2-0}-q_{2-0}+\frac{1}{2} V_{2}^{2} \\
& =\frac{1}{k-1} R\left(T_{2}-T_{0}\right)+\frac{1}{2} V_{2}^{2} \\
& =\frac{p_{\mathrm{a}}}{k-1}\left(\frac{1}{\rho_{2}}-\frac{1}{\rho_{\mathrm{a}}}\right)+\frac{1}{2} V_{2}^{2}
\end{aligned}
$$

Energy equation for Newtonian ideal airflow. The airflow experiences a steady laminar flow process of fully developed viscous incompressible fluid in the process 1 , the kinetic energy of the unit mass of airflow at section A equals to that at section $\mathrm{B}$, that is

$$
\frac{1}{2} V_{1}^{2}=\frac{1}{2} V_{2}^{2}
$$

Add the $e_{1-2}$ and $e_{2-0}$ together, gives $e_{1-0}$, the total energy loss of the unit mass of airflow from the initial state, the state 1 , to the reference state, the state 0 :

$$
\begin{aligned}
& e_{1-0}=e_{1-2}+e_{2-0} \\
& =\frac{k}{k-1} \frac{p_{\text {guage }}}{\rho_{1}}+\frac{1}{k-1} R\left(T_{2}-T_{0}\right)
\end{aligned}
$$

$$
\begin{aligned}
& e_{1-0}=\frac{p_{1}^{*}-p_{\mathrm{a}}}{\rho_{1}}+\frac{1}{k-1} \frac{p_{1}^{*}-p_{\mathrm{a}}}{\rho_{1}}+\frac{1}{k-1} R\left(T_{2}-T_{0}\right) \\
& =\frac{p_{1}^{*}-p_{\mathrm{a}}}{\rho_{1}}+\frac{1}{k-1} R\left(T_{1}^{*}-T_{2}\right)+\frac{1}{k-1} R\left(T_{2}-T_{0}\right) \\
& =\frac{p_{1}^{*}-p_{\mathrm{a}}}{\rho_{1}}+\mathrm{c}_{\mathrm{v}}\left(T_{1}^{*}-T_{0}\right)
\end{aligned}
$$

That is

$$
e_{1-0}=\frac{p_{1}^{*}-p_{\mathrm{a}}}{\rho_{1}}+\mathrm{c}_{\mathrm{v}}\left(T_{1}^{*}-T_{0}\right)
$$

The above equation is the energy equation for Newtonian ideal airflow.

It can be seen that the energy of Newtonian ideal airflow include the potential energy caused by its pressure deviated from the reference pressure, the potential energy of pressure, and the potential energy caused by its temperature deviated from the reference temperature, the internal energy.

Taking $q_{m}$ present the mass flow of the airflow at section A, the total energy taken away by the airflow per unit time $E_{1-0}$ is

$$
E_{1-0}=q_{\mathrm{m}} e_{1-0}=q_{\mathrm{m}}\left[\frac{p_{1}^{*}-p_{\mathrm{a}}}{\rho_{1}}+c_{\mathrm{v}}\left(T_{1}^{*}-T_{0}\right)\right]
$$

It's important to note that the applying premise of the energy equation for Newtonian ideal airflow is a Newton ideal airflow of low velocity (Mach number less than 0.3 , that is, the compressibility of the airflow can be neglected), steady and laminar flow.

\section{Quantitative research on gas exchange losses}

In practical cycles of an internal combustion engines, the main reason for the irreversible losses include the time loss caused by a finite burning speed of air-fuel mixture, the loss caused by the properties of working medium, the loss caused by heat transfer and the loss caused by gas exchange. The power loss of the practical cycles $\mathrm{Pa}$ can be expressed as

$$
P_{\mathrm{a}}=P_{\mathrm{r}}+P_{\mathrm{c}}+P_{\mathrm{s}}+P_{\mathrm{w}}
$$

Where the $P_{r}$ represents the power loss caused by the gas exchange; the $P_{c}$ represents the power loss caused by the heat transfer; the $P_{s}$ represents the power loss caused by the finite burning speed of air-fuel mixture; the $P_{w}$ represents the power loss caused by the properties of working medium.

In practical cycles of an internal combustion engines, the internal friction power loss $P_{f}$ is all converted into heat and taken away by the cooling medium such as the coolant and oil so the power balance equation of the practical cycles can be expressed as

$$
\begin{aligned}
& P_{\mathrm{tcb}}=P_{\mathrm{t}} \eta_{\mathrm{cb}} \\
& =P_{\mathrm{e}}+P_{\mathrm{f}}+P_{\mathrm{r}}+P_{\mathrm{c}}+P_{\mathrm{s}}+P_{\mathrm{w}}
\end{aligned}
$$

Where the $P_{t}$ represents the theoretical power; the $P_{t c b}$ represents the theoretical power which take the combustion efficiency $\eta_{c b}$ into account; the $P_{e}$ represents the effective output power.

The above equation can be simplified as 


\subsection{Test device}

Figure 2 shows the layout of engine test bench. The engine bench test was carried out to a naturally aspirated port injection LJ491QE1 gasoline engine. The primary parameters of the engine are listed as follows: the displacement is 2.237 $\mathrm{L}$, the compression ratio is 8.8 , the maximum torque (speed) is $190 \mathrm{~N} \cdot \mathrm{m}(2400 \mathrm{r} / \mathrm{min}-2800 \mathrm{r} / \mathrm{min})$, the maximum power (speed) is $75.0 \mathrm{~kW}(4600 \mathrm{r} / \mathrm{min})$. The sensors series for collecting the engine working state parameters include the coolant temperature sensor, the intake air temperature \& pressure sensor, the exhaust temperature sensor and the exhaust pressure sensor, etc.

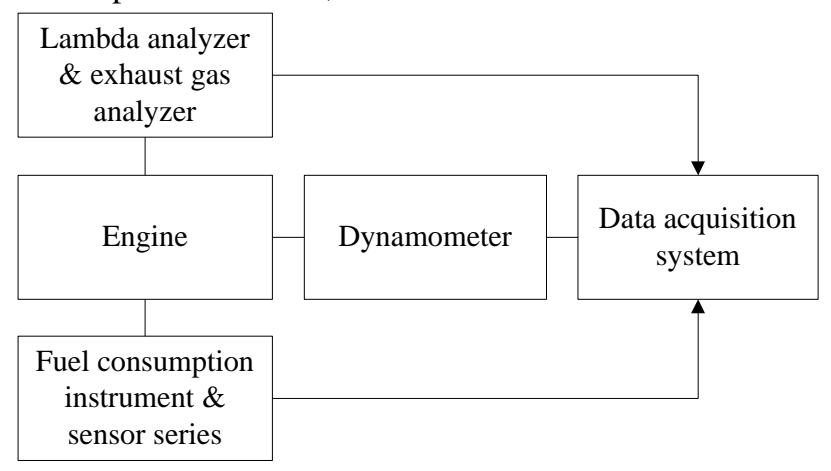

Figure 2: Layout of the engine test bench

\subsection{Quantitative research on the intake loss of LJ491QE1 gasoline engine}

In order to analysis the power loss caused by the intake process of a naturally aspirated port fuel injection gasoline engine, the intake process of the engine should be simplified reasonably. The simplification include that the intake is a homogeneous mixture of air and fuel, and the intake process is a steady state steady flow process of an open system.

Gasoline engine usually use an integrated sensor mounted in the intake tube between the throttle and intake manifold to measure the intake temperature and pressure. The propagation velocity of the pressure can reach more than hundreds of meters per second ${ }^{[2]}$ and the inner surface of the intake tube between the position where the pressure is measured and the intake valve is smooth without parts that could cause local resistance loss. If the route loss of the inner surface of the intake tube between the position where the pressure is measured and the intake valve is ignored and take the entropy generation caused by the choking effect of the intake valve account into the power loss caused by heat transfer $P_{c}$, the intake parameters measured by the intake temperature and pressure sensor can be used to determine the state of intake and quantitatively calculate the intake power loss $P_{i n}$.

According to the universal characteristics test data of the LJ491QE1 engine, the maximum Reynolds number of intake was 59, under the engine operating state of $4200 \mathrm{r} / \mathrm{min}$ and full load. The maximum Reynolds number is much less than the critical Reynolds number 2320, which is used to determine the airflow state in engineering applications, so the intake flow at section of the intake tube where the intake temperature and pressure was measured formed a laminar flow state. The maximum value of intake Mach number was
0.025 , much less than 0.3 , under the engine operating state of 4200 r/min, 90\% load (computing method for the Reynolds number and Mach number can be found in the reference [1]). In conclusion, the intake state met the application condition of the energy equation for Newtonian ideal airflow, and the intake power loss Pin of the engine can be calculated by the equation.

Based on the energy equation for Newtonian ideal airflow, the energy loss of unit mass of intake caused by air suction of the LJ491QE1 engine can be expressed as

$$
e_{\mathrm{i}}=\frac{p_{\mathrm{i}}^{*}-p_{\mathrm{a}}}{\rho_{\mathrm{i}}}+c_{\mathrm{vi}}\left(T_{\mathrm{i}}^{*}-T_{0}\right)
$$

Where the $p_{i}{ }^{*}$ represents the total pressure of intake, the $\rho_{i}$ represents the density of intake, the $T_{i}^{*}$ represents the total temperature of intake, the $c_{v i}$ represents the specific heat at constant volume of intake (computing method of the $c_{v i}$ can be found in the reference [1]).

According to the universal characteristics test data of the LJ491QE1 engine, the heating effect by the intake tube to the intake airflow was more distinct under the engine operating state of light load and low speed than under the engine operating state of heavy load and high speed, and the maximum temperature rise of the intake airflow was 10 degrees Celsius comparing with the atmospheric temperature $T_{0}$. Under the engine operating state of theoretical air-fuel ratio, by calculation, when the temperature of unit mass of the intake airflow rise 10 degrees Celsius, the increment of its thermal energy only account for 0.246 percent of the thermal energy released by the air-fuel mixture in combustion chambers of the engine ${ }^{[1]}$, so the in the above equation which represents the thermal energy of unit mass of intake airflow caused by its temperature deviated from the reference state can be ignored, that is, the energy loss of unit mass of intake caused by air suction can be expressed as

$$
e_{\mathrm{i}}=\frac{p_{\mathrm{vi}}}{\rho_{\mathrm{i}}}
$$

Where the $p_{v i}$ represents the vacuum pressure of intake, and if use $q_{m i}$ represents mass flow rate of the intake, the intake loss power $P_{i n}$ can be expressed as

$$
P_{\text {in }}=q_{\mathrm{mi}} \frac{p_{\mathrm{vi}}}{\rho_{\mathrm{i}}}
$$

Figure 3 shows the effective output power $P_{e}$ and the intake loss power $P_{i n}$ as functions of load percentage $l_{p}$, ratio between the engine load and the maximum engine load at the same engine speed, under $1800 \mathrm{r} / \mathrm{min}$ load characteristics tests of the LJ491QE1 engine. It can be seen that the intake loss power $P_{i n}$ and the load percentage $l_{p}$ have an inverse relationship. Based on analysis of the tests results, the intake loss power $P_{\text {in }}$ accounts for 63.9 percent of the effective output power $P_{e}$ when the load percentage $l_{p}$ is 0.1 , the $P_{\text {in }}$ accounts for 7.5 percent of the $P_{e}$ when the $l_{p}$ is 0.5 and the $P_{i n}$ accounts for 0.1 percent of the $P_{e}$ when the $l_{p}$ is 1 .

Figure 4 shows the effective output power $P_{e}$ and the intake loss power $P_{\text {in }}$ as functions of the load percentage $l_{p}$, under the $3800 \mathrm{r} / \mathrm{min}$ load characteristics tests of LJ491QE1 engine. It can be seen that the $P_{i n}$ and the $l_{p}$ also have an inverse relationship. Based on analysis of the tests results, the $P_{\text {in }}$ 
accounts for 56.2 percent of the $P_{e}$ when the $l_{p}$ is 0.1 , the $P_{i n}$ accounts for 8.1 percent of the $P_{e}$ when the $l_{p}$ is 0.5 , and the $P_{i n}$ accounts for 0.3 percent of the $P_{e}$ when the $l_{p}$ is 1 .

Given the above, under the load characteristics tests of the naturally aspirated port fuel injection gasoline engine, the ratio between the intake loss power $P_{\text {in }}$ and effective output power $P_{e}$ decreases with the load percentage $l_{p}$ increasing, and the intake loss power $P_{\text {in }}$ make up a larger percentage of the effective output power $P_{e}$ under the engine operating states of light load.

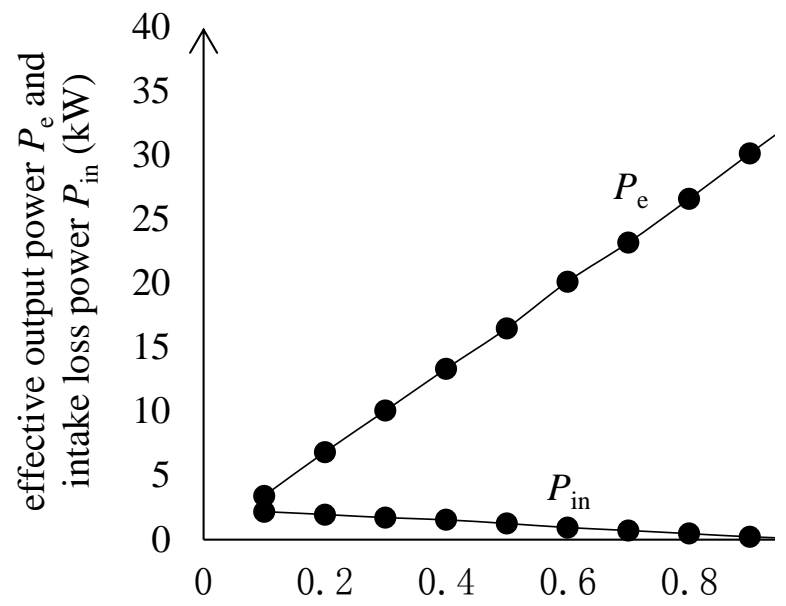

Figure 3: Effective output power $P_{e}$ and intake loss power $P_{\text {in }}$ as functions of load percentage $l_{p}$ under $1800 \mathrm{r} / \mathrm{min}$ load characteristics tests of the LJ491QE1 engine

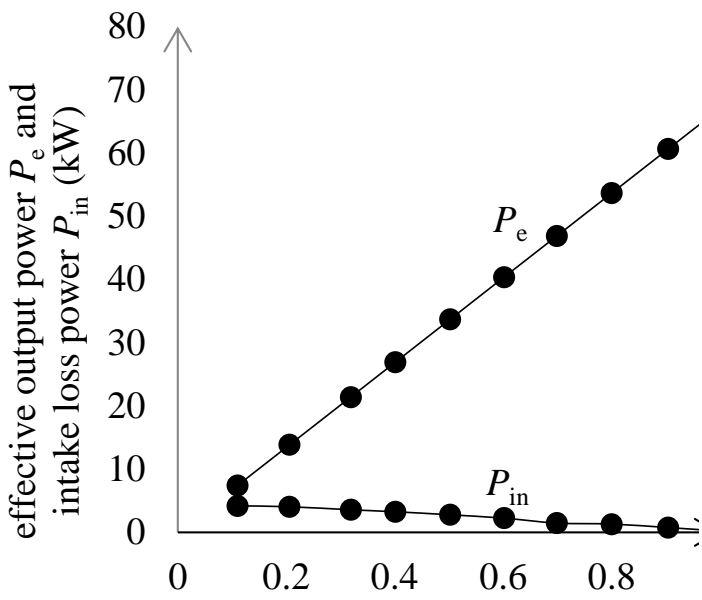

Figure 4: Effective output power $P_{e}$ and intake loss power $P_{\text {in }}$ as functions of load percentage $l_{p}$ under $3800 \mathrm{r} / \mathrm{min}$ load characteristics tests of the LJ491QE1 engine

According to the national standard, the spacing between downstream exit of the exhaust manifold and the measuring position of the exhaust pressure on the exhaust pipe is $75 \mathrm{~mm}$, and the spacing between the measuring positions of the exhaust pressure and the exhaust temperature is $25 \mathrm{~mm}{ }^{[4]}$, where the radius and the curvature radius of the exhaust pipe are large enough to ignore the route loss and the local loss, and the two measuring positions can be considered as the same section of the exhaust pipe, so the exhaust state parameters measured by the exhaust temperature and pressure sensors can be used to determine the exhaust state and calculate the exhaust power loss $P_{e x}$ quantitatively.

According to the universal characteristics test data of the LJ491QE1 engine, the maximum Reynolds number of the exhaust was 83 , under the rated operating state of the engine (4600 r/min, full load), and the maximum Reynolds number is much less than the critical Reynolds number 2320 which is used to determine air flow states in engineering applications, so the exhaust flow at the sections of the exhaust pipe where the exhaust temperature and pressure were measured formed a laminar flow state. The maximum Mach number of the exhaust was 0.102 , much less than 0.3 ; also under the rated operating state of the engine (computing method of the Reynolds number and Mach number can be found in the reference [3]). In conclusion, the exhaust states meet the application condition of the energy equation for Newtonian ideal airflow, and the exhaust power loss $P_{e x}$ of the engine can be calculated by the equation.

Based on the energy equation for Newtonian ideal airflow, the energy taken away by the unit mass of exhaust $e_{e}$ can be expressed as

$$
e_{\mathrm{e}}=\frac{p_{\text {guage }}}{\rho_{\mathrm{e}}}+c_{\mathrm{ve}}\left(T_{\mathrm{e}}^{*}-T_{0}\right)
$$

Where the $p_{\text {gauge }}$ represents the gauge pressure of the exhaust, $\rho_{e}$ represents the density of the exhaust, $T_{e}{ }^{*}$ represents the total temperature of the exhaust, $c_{v e}$ represents the specific heat at constant volume of the exhaust (computing method of the $c_{v e}$ and the gas constant of the exhaust $R_{g e}$ can be found in the reference [3]).

It can be seen from the above equation that the energy of unit mass of the exhaust $e_{e}$ include two parts of the $p_{\text {guage }} / \rho_{e}$ and the $c_{v e}\left(T_{e}{ }^{*}-T_{0}\right)$. The $p_{\text {guage }} / \rho_{e}$ is the energy loss caused by the exhaust valve opened ahead of schedule, named as the expansion loss, and caused by the exhaust pushed forcibly out of the cylinders by the pistons, named as the push out loss. The $p_{\text {guage }} / \rho_{e}$ is the useful energy, it is also the ordering energy, and it can all be converted into flow work. That is to say, for unit mass of the exhaust, on the basis of the energy equation for Newtonian ideal airflow, the potential energy caused by its pressure deviated from the reference pressure is equal to the sum of the expansion loss and the push out loss of the unit mass of the air-fuel mixture. The $c_{v e}\left(T_{e}{ }^{*}-T_{0}\right)$ is the thermal energy of unit mass of the air-fuel mixture after thermal cycle of the engine. Regardless of the potential energy caused by the exhaust pressure, the thermal energy caused by the exhaust temperature deviated from the reference temperature can't be converted into work without heat engine.

Analysing in the view of flow, in response to the push out work at the forced exhaust stage and the expansion work at the free exhaust stage, the energy of unit mass of the exhaust flow is increased from the thermal energy $c^{e}{ }_{v 0} \cdot T_{e}^{*}$ to the enthalpy $c_{p 0}^{e} \cdot T_{e}^{*}$, and the difference between the enthalpy $c_{p 0}^{e} \cdot T_{e}^{*}$ and the thermal energy $c_{v 0}^{e} \cdot T_{e}^{*}, R_{g e}$, is the sum of the push out work at the forced exhaust stage and the expansion work at the free exhaust stage of the exhaust stroke. The energy increment $R_{g e}$ is the useful energy, the ordering energy and it can all be converted into flow work. 
Given the above, the exhaust loss $P_{e x}$ is

$$
P_{\text {ex }}=q_{\mathrm{mi}} \frac{p_{\text {gage }}}{\rho_{\mathrm{e}}}
$$

Figure 5 shows the effective output power $P_{e}$ and the exhaust loss power $P_{e x}$ as functions of the load percentage $l_{p}$ under the $2200 \mathrm{r} / \mathrm{min}$ load characteristics tests of the LJ491QE1 engine. It can be seen that the exhaust loss power $P_{e x}$ and the load percentage $l_{p}$ have a proportional relationship. Based on analysis of the test result, the exhaust loss power $P_{e x}$ accounts for 1.7 percent of the effective output power $P_{e}$ when the load percentage $l_{p}$ is 0.1 , the $P_{e x}$ accounts for 2 percent of the $P_{e}$ when the $l_{p}$ is 0.5 and the $P_{e x}$ accounts for 5 percent of the $P_{e}$ when the $l_{p}$ is 1 .

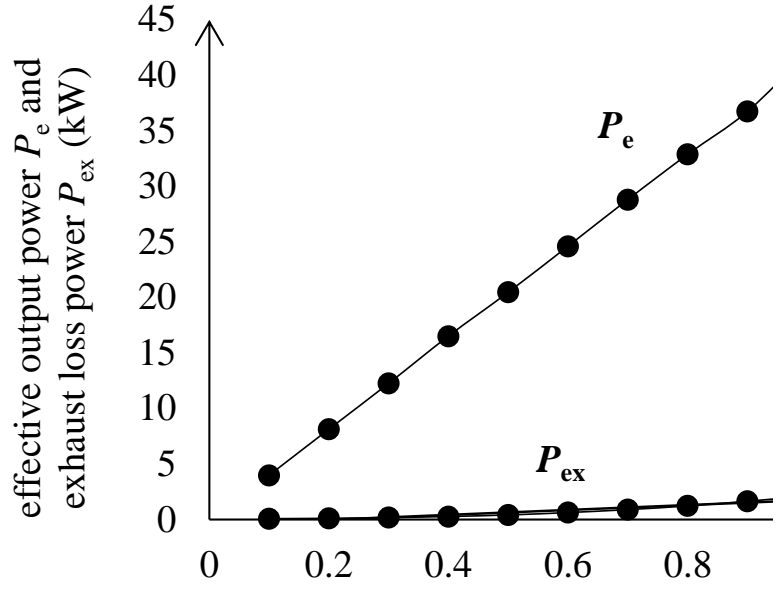

Figure 5: Effective output power $P_{e}$ and exhaust loss power $P_{e x}$ as functions of the load percentage $l_{p}$ under 2200 $\mathrm{r} / \mathrm{min}$ load characteristics tests of the LJ491QE1 engine

Figure 6 shows the effective output power $P_{e}$ and the exhaust loss power $P_{e x}$ as functions of the load percentage $l_{p}$ under the $4200 \mathrm{r} / \mathrm{min}$ load characteristics tests of the LJ491QE1 engine. It can be seen that the exhaust loss power $P_{e x}$ and the load percentage $l_{p}$ also have a proportional relationship. Based on analysis of the test result, the exhaust loss power $P_{e x}$ accounts for 4.9 percent of the effective output power $P_{e}$ when the load percentage $l_{p}$ is 0.1 , the $P_{e x}$ accounts for 7.2 percent of the $P_{e}$ when the $l_{p}$ is 0.5 and the $P_{e x}$ accounts for 13.9 percent of the $P_{e}$ when the $l_{p}$ is 1 .

Given the above, under the load characteristics tests of the naturally aspirated port fuel injection gasoline engine, the ratio between the exhaust loss power $P_{e x}$ and effective output power $P_{e}$ increase with increasing the load percentage $l_{p}$, and the exhaust loss power $P_{e x}$ make up a larger percentage of the effective output power $P_{e}$ under heavy load engine states.

\section{Summary and conclusions}

The concept of the Newton ideal airflow was defined, and the energy equation for Newtonian ideal airflow was deduced in theory, which can be used to quantitatively calculate the gas exchange loss of internal combustion engine. Experimental research was also conducted by employing the naturally aspirated port fuel injection LJ491QE1 gasoline engine and the gas exchange loss of the engine was calculated quantitatively by using the energy equation for Newtonian ideal airflow. The results shows that the ratio between the intake loss power $P_{i n}$ and the effective output power $P_{e}$ decrease with increasing the load percentage $1 \mathrm{p}$, and the intake loss power $P_{\text {in }}$ make up a larger percentage of the effective output power $P_{e}$ under light load engine states, the ratio between the exhaust loss power $P_{e x}$ and effective output power $P_{e}$ increase with increasing the load percentage $l_{p}$, and the exhaust loss power $P_{e x}$ make up a larger percentage of the effective output power $P_{e}$ under heavy load engine states.

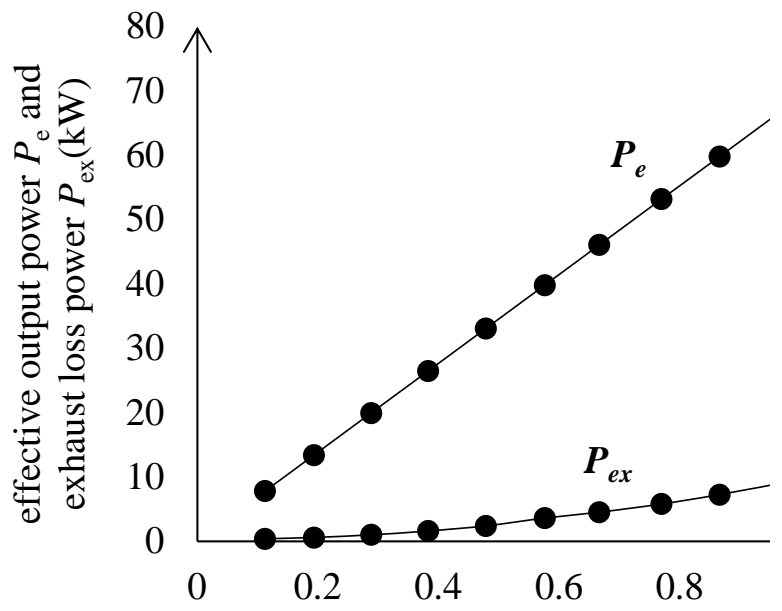

Figure 6. Effective output power $P_{e}$ and exhaust loss power $P_{e x}$ as functions of the load percentage $l_{p}$ under 4200 $\mathrm{r} / \mathrm{min}$ load characteristics tests of the LJ491QE1 engine

\section{Acknowledgements}

This work is supported by Liaoning Provincial Committee of Education General Research Projects (No.L2014594), all support is gratefully acknowledged.

\section{References}

[1] Wang Xinyue, Gas Dynamics Fundamental, Xi'an: Northwestern Polytechnic University Press, 2006: 93-123.

[2] Hua Ziqiang, Zhang Zhongjin, Engineering Thermodynamics, Beijing: Higher Education Press, 2000: 7799.

[3] Liang Jinguang, Study on Efficiency Model of Gasoline Engine Used by Hybrid Electrical Vehicle, Doctoral Dissertations of Jilin University, 2010: 49-66.

[4] China State Bureau of Quality and Technical Supervision (CSBTS), GB 17691-2001 Limits and measurement methods for exhaust pollutants from compression ignition (C.I.) engines of vehicles, Beijing: China Standards Press, 2001: 6.

[5] Bosch Company (Germany), Gasoline Engine Management, translated by Wu Sen, Beijing: Beijing Institute Technology Press, 2009. 